\title{
Transfemoral versus transapical approach for transcatheter aortic valve implantation: hospital outcome and risk factor analysis.
}

\author{
Enrico Ferrari ${ }^{1,2^{*}}$, Eric Eeckhout ${ }^{3}$, Sanjiv Keller ${ }^{2}$, Olivier Muller ${ }^{3}$, Piergiorgio Tozzi ${ }^{2}$, Denis Berdajs ${ }^{4}$ \\ and Ludwig Karl von Segesser ${ }^{2}$
}

\begin{abstract}
Background: Transcatheter aortic valve implantation is indicated in high-risk patients with aortic stenosis. We compared the clinical outcome of 180 consecutive patients who underwent transapical (TA) and transfemoral (TF) procedures in a single centre.

Methods: Ninety consecutive TA (TA-group) and 90 consecutive TF (TF-group) were performed from 2009 to 2014. Clinical variables were prospectively collected and retrospectively analysed for hospital outcomes and to identify risk factors for hospital mortality, vascular complications and stroke.

Results: Mean age was $80 \pm 8.5$ and $83 \pm 8.4$ years, in the TA and TF-group, respectively. TA-group presented higher prevalence of comorbidities: more vascular disease (79\% vs 22\%, $p<0.001$ ), chronic pulmonary disease (32\% vs $10 \%, p<0.001)$, previous vascular surgery ( $14 \%$ vs $4 \%, p=0.039)$, coronary disease $(60 \%$ vs $40 \%, p=0.007)$, and previous cardiac surgery ( $28 \%$ vs $17 \%, p=0.073$ ). Logistic Euroscore was $36 \pm 15 \%$ in the TA-group and $25 \pm 14 \%$ in the TF-group ( $p<0.001$ ), but hospital mortality was similar (TA:9\%, TF:10\%, $p=0.799$ ). Access-related vascular complications occurred more often in transfemoral patients (TA:3\%, TF:11\%, $p=0.081$ ) while major bleeding (TA:3\%, TF:4\%, $p=1$ ) and stroke (TA:2\%, TF:3\%, $p=1$ ) were equally distributed. Postoperative renal failure and dialysis were associated with impaired neurological outcome ( $p=0.035$ and $p=0.020$, respectively). Mild to severe paravalvular leak was more prevalent in transfemoral patients (TA:5\%, TF:25\%, $p<0.001$ ).
\end{abstract}

Conclusions: In our experience, the TA and TF-group presented different risk profiles but mortality rate and adverse neurological outcome had a similar incidence. The transfemoral approach carried more vascular complications and paravalvular leaks but last-generation devices will improve this outcome.

Keywords: Transcatheter aortic valve implantation, Aortic valve stenosis, Transfemoral aortic valve implantation, Transapical aortic valve implantation

\section{Background}

Aortic stenosis is the most common acquired heart valve disease in the adult and the surgical aortic valve replacement (SAVR) remains the treatment of choice with good outcomes and long-term results [1-4]. However, patients with comorbidities carry a higher surgical risk and, therefore, they might benefit from recently developed

\footnotetext{
* Correspondence: enricoferrari@bluewin.ch

${ }^{1}$ Department of Cardiac Surgery, Cardiocentro Ticino Foundation, Via Tesserete 48, 6900 Lugano, Switzerland

${ }^{2}$ Cardiovascular Research Unit, University Hospital, Lausanne, Switzerland Full list of author information is available at the end of the article
}

minimally invasive techniques and technologies. Since 2007, the transcatheter aortic valve implantation (TAVI) has become a widely-accepted alternative to open heart surgery in patients with high-risk profiles and, so far, more than $200^{\prime} 000$ procedures have already been performed worldwide, mostly with the CoreValve ${ }^{\mathrm{Tm}}$ (Medronic, Minneapolis, MN) and the SAPIEN ${ }^{\mathrm{mi}}$ (Edwards Lifesciences, Irvine, CA) valve families with good hospital and mid-term results [5-8]. The two arms of the PARTNER trial have proven the safety and efficacy of TAVI in inoperable and high-risk patients with superior results when compared to medical treatment and non-inferior 
results when compared to standard surgery [7, 8]. Moreover, recently published studies have also shown good valve hemodynamic parameters in early and mid-term follow-ups and in intermediate-risk patients [9-15].

Alternative access routes have been explored but the two most popular are still the transapical (TA) and the transfemoral (TF): however, patient attribution to these accesses is still questionable in absence of a severe peripheral vascular disease. We compared the outcome of our first 180 consecutive TA and TF TAVI patients and we identified risk factors for postoperative hospital mortality, vascular complications and stroke.

\section{Methods}

In the database, we identified the first 90 TA-TAVI cases (TA-group) and the first 90 TF-TAVI cases (TF-group) performed from 2009 to 2014. In our institution, TATAVI was performed since 2009 while TF-TAVI were scheduled since 2010. During the same period of time, only few transaortic TAVI have been performed and they are not included in the study.

Clinical variables were prospectively collected in the hospital database and then retrospectively analysed to compare the two groups and to identify risk factors for hospital mortality (30 days mortality or within hospital stay), neurological events (stroke) and vascular complications (major vascular complications including accesssite related complications, aortic dissections, aortic root rupture and tamponade due to ventricular rupture) using the VARC-2 definitions described elsewhere [16]. All patients signed informed consents for the index procedure, for the use of clinical data in medical research and for clinical data analysis (Patients included in the Swiss TAVI Registry approved by the Ethics Committee of the Vaud State in Switzerland).

\section{Patients selection criteria}

Patients suffering from severe symptomatic aortic valve stenosis with concomitant comorbidities were studied for inclusion in the TAVI program. Standard inclusion criteria for TAVI were used to identify good candidates and the Logistic Euro-SCORE was calculated for all patients to predict hospital mortality. In order to proceed with transcatheter interventions the final decision came from the hospital Heart-Team, in particular concerning patients not fulfilling standard criteria (i.e. younger patients with severe liver disease or patients with porcelain aortas).

Important exclusion criteria for both TA and TF approach were the presence of concomitant severe valvular or coronary disease not suitable for percutaneous procedure, severe left ventricular dysfunction with ejection fraction below $20 \%$ and severe patient frailty evaluated by the Heart-Team, which consists of a cardiologist, a surgeon (both coordinators for the TAVI program), an anaesthesiologist, a radiologist and a geriatrician.

An important observation is that during the study period, transapical and transfemoral procedures were not equally distributed (Fig. 1). This was due to the access-site selection process performed by the HeartTeam that embraced, during the years, the launch of new "low-profile" transfemoral devices leading to more valves implanted transfemorally.

All patients enrolled in the TAVI program underwent complementary trans-thoracic echocardiogram, coronary angiogram and three-dimensional cardiovascular computed tomography scan (3D CT-scan) to analyse alternative access routes and to determine the valve size. Peripheral vascular access routes smaller than $6 \mathrm{~mm}$ diameter or presenting severe annular calcifications were not considered good access sites for transfemoral TAVR, such as the presence of abdominal aorta aneurysms and vessel tortuosity. In these cases, TA was considered the best option. Predilation of small ilio-femoral arteries was never attempted. The aortic valve annulus was assessed by injected aortic CT-scan and trans-oesophageal echocardiography using the diameter, the area and the perimeter for the stent-valve sizing. Patients with chronic kidney failure underwent CTscan and coronary angiograms with low-dose of contrast.

\section{Ta-Tavi}

TA-TAVI were performed under general anaesthesia through a left antero-lateral mini-thoracotomy at fifth intercostal space. Intraoperative imaging included transoesophageal echocardiography to confirm the valve size and fluoroscopy. The apexes were prepared with concentric reinforced purse-string sutures and the devices were the Sapien ${ }^{\text {Tw }}(2009-2010)$, the Sapien ${ }^{\text {Tw }}$ XT (20112013) and the Sapien ${ }^{\text {tux }} 3$ (2014) valves (Edwards Lifesciences, Irvine, CA). At the beginning of our series, complication-free patients used to be transferred intubated to the intensive care unit while, after getting more experienced, patients were rapidly extubated in the cathlab and transferred to the intermediate care unit. As previously reported, echo-guided TA-TAVI were performed without contrast injections [17].

\section{Tf-Tavi}

Patients with good vascular access underwent TF-TAVI under general anaesthesia. According to our policy, the vascular access was surgically performed with a $3 \mathrm{~cm}$ skin incision at the groin in order to puncture the femoral artery under direct vision and prevent collateral damage. Cardiac imaging was similar to TA-TAVI while the devices were the Sapien $^{\text {tx }}$ valves, as mentioned above, but also the Medtronic CoreValve $^{\mathrm{Tm}}$ (Medtronic inc, Minneapolis, MN). All complication-free patients were extubated in the cath-lab and transferred to the intermediate care unit. 


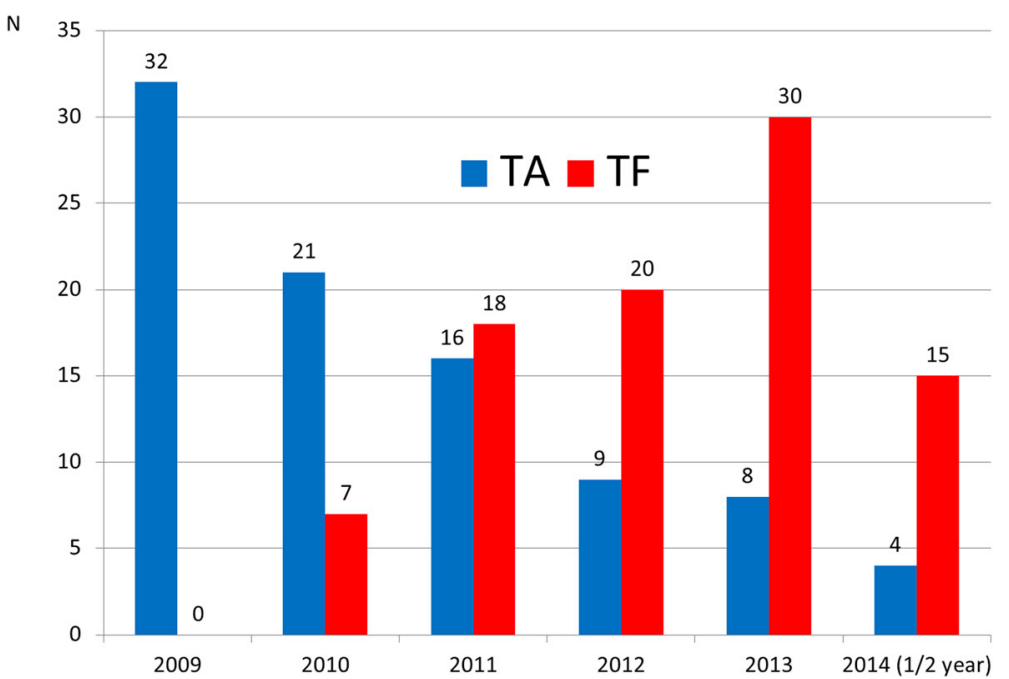

Fig. 1 Transapical and transfemoral TAVI distribution in our hospital during the study period

\section{Statistical analysis}

Statistical analysis was performed using R (version 3.2.2). Continuous variables are summarized as mean \pm Standard Deviation and a t-test is used to compare the two groups. Categorical variables are presented as numbers and proportions (\%) and a $\chi^{2}$ test or a Fisher exact test is used to compare the two groups. Selected categorical and continuous variables were analysed as risk-factors for hospital mortality (death occurring within 30 days or during the same hospitalization), disabling neurological

Table 1 Demographics, symptoms, risk factors

\begin{tabular}{|c|c|c|c|c|}
\hline & $\begin{array}{l}\text { Overall } \\
(N=180)\end{array}$ & $\begin{array}{l}\text { TA-TAVI } \\
(N=90)\end{array}$ & $\begin{array}{l}\text { TF-TAVI } \\
(N=90)\end{array}$ & $p$ \\
\hline Mean age (years) & $82 \pm 8.6$ & $80 \pm 8.5$ & $83 \pm 8.4$ & 0.014 \\
\hline Men & $82(46 \%)$ & $45(50 \%)$ & $37(41 \%)$ & $0.231^{\mathrm{a}}$ \\
\hline COPD & $38(21 \%)$ & $29(32 \%)$ & $9(10 \%)$ & $<0.001^{\mathrm{a}}$ \\
\hline Peripheral vascular disease & $91(51 \%)$ & $71(79 \%)$ & $20(22 \%)$ & $<0.001^{\mathrm{a}}$ \\
\hline Previous vascular surgery & $17(9 \%)$ & $13(14 \%)$ & $4(4 \%)$ & 0.039 \\
\hline Coronary disease & $90(50 \%)$ & $54(60 \%)$ & $36(40 \%)$ & $0.007^{\mathrm{a}}$ \\
\hline Previous coronary surgery & $27(15 \%)$ & $19(21 \%)$ & $8(9 \%)$ & $0.022^{\mathrm{a}}$ \\
\hline Previous cardiac surgery & $40(22 \%)$ & $25(28 \%)$ & $15(17 \%)$ & $0.073^{\mathrm{a}}$ \\
\hline Previous coronary angioplasty/stenting & $28(16 \%)$ & $13(14 \%)$ & $15(17 \%)$ & $0.681^{\mathrm{a}}$ \\
\hline Hypertension & $117(65 \%)$ & $56(62 \%)$ & $61(68 \%)$ & $0.435^{\mathrm{a}}$ \\
\hline Chronic renal failure & $75(42 \%)$ & $38(42 \%)$ & $37(41 \%)$ & $0.880^{\mathrm{a}}$ \\
\hline Dialysis & $7(4 \%)$ & $4(4 \%)$ & $3(3 \%)$ & 1 \\
\hline Previous stroke & $20(11 \%)$ & $11(12 \%)$ & $9(10 \%)$ & $0.635^{\mathrm{a}}$ \\
\hline Diabetes (insulin) & $30(17 \%)$ & $18(20 \%)$ & $12(13 \%)$ & $0.230^{\mathrm{a}}$ \\
\hline Liver disease (CHILD score) & $5(3 \%)$ & $3(3 \%)$ & $2(2 \%)$ & 1 \\
\hline pacemaker implantation & $21(12 \%)$ & $9(10 \%)$ & $12(13 \%)$ & $0.486^{\mathrm{a}}$ \\
\hline Chest radiotherapy & $16(9 \%)$ & $7(8 \%)$ & $9(10 \%)$ & $0.600^{\mathrm{a}}$ \\
\hline Preoperative critical state & $15(8 \%)$ & $13(14 \%)$ & $2(2 \%)$ & 0.005 \\
\hline Porcelain aorta & $12(13 \%)$ & $12(13 \%)$ & $0(0 \%)$ & 1 \\
\hline logistic Euro-SCORE (\%) & $31 \pm 16$ & $36 \pm 15$ & $25 \pm 14$ & $<0.001$ \\
\hline
\end{tabular}

Data presented as mean $\pm \mathrm{SD}$ or $\mathrm{N}(\%)$

${ }^{a}$ Chi2 value, otherwise it's a Fischer test or a T-Test value

COPD Chronic Obstructive Pulmonary Disease 
Table 2 Preoperative imaging and valve hemodynamic

\begin{tabular}{|c|c|c|c|c|}
\hline & $\begin{array}{l}\text { Overall } \\
(N=180)\end{array}$ & $\begin{array}{l}\text { TA-TAVI } \\
(N=90) \\
\end{array}$ & TF-TAVI $(N=90)$ & $\mathrm{p}$ \\
\hline Transaortic valve gradient $(\mathrm{mmHg})$ & $67 \pm 26$ & $62 \pm 25$ & $71 \pm 28$ & \\
\hline Mean aortic valve area $\left(\mathrm{cm}^{2}\right)$ & $0.9 \pm 3.4$ & $1.2 \pm 4.8$ & $0.7 \pm 0.2$ & \\
\hline Indexed aortic valve area $\left(\mathrm{cm}^{2} / \mathrm{m}^{2}\right)$ & $0.4 \pm 0.1$ & $0.4 \pm 0.1$ & $0.4 \pm 0.1$ & \\
\hline Mean left ventricular ejection fraction (\%) & $54 \pm 13$ & $52 \pm 12$ & $56 \pm 13$ & \\
\hline$>50 \%$ & $101(56 \%)$ & $42(47 \%)$ & $59(66 \%)$ & $0.008^{*}$ \\
\hline $30-50 \%$ & $70(39 \%)$ & $42(47 \%)$ & $28(32 \%)$ & \\
\hline$<30 \%$ & $8(4 \%)$ & $6(6 \%)$ & $2(2 \%)$ & \\
\hline Pulmonary hypertension & $101(56 \%)$ & $48(53 \%)$ & $53(59 \%)$ & $0.453^{*}$ \\
\hline Mean aortic annulus diameter measured with CT-scan (mm) & $23 \pm 2.3$ & $23 \pm 2.5$ & $24 \pm 2.1$ & \\
\hline Mean aortic annulus diameter measured with TOE (mm) & $23 \pm 2.2$ & $22 \pm 2$ & $22 \pm 2.4$ & \\
\hline Mean distance: annulus-left coronary ostium (mm) & $13 \pm 2.9$ & $12 \pm 2$ & $14 \pm 3.2$ & \\
\hline Mean distance: annulus-right coronary ostium (mm) & $134 \pm 4$ & $12 \pm 3$ & $15 \pm 4.4$ & \\
\hline
\end{tabular}

Data presented as mean \pm SD or $\mathrm{N}(\%)$. ${ }^{*}$ Chi2 value

CT Computed Tomography, TOE Transoesophageal echocardiography

complications and major vascular complications, using univariate logistic regression. A $p$-value below 0.05 was considered statistically significant.

\section{Results}

Baseline clinical characteristics are described in Tables 1 and 2. Mean age was $80 \pm 8.5$ and $83 \pm 8.4$ years, in the TA and TF-group, respectively $(p=0.014)$, while the sex distribution was similar $(50 \%$ male patients in the TAgroup and $41 \%$ in the TF-group, $p=0.231$ ).

TA-group presented higher prevalence of comorbidities: more vascular disease (TA:79\%, TF:22\%, $p<0.001$ ), chronic pulmonary disease (TA:32\%, TF:10\%, $p<0.001$ ), higher prevalence of previously performed vascular surgery (TA:14\%, TF:4\%, $p=0.039$ ), higher coronary disease (TA:60\%, TF:40\%, $p=0.007$ ), more previously performed coronary surgery (TA:21\%, TF:9\%, $p=0.022$ ) and more previously performed cardiac surgery (TA:28\%, TF:17\%, $p=0.073$ ). Thirteen percent of patients in the TA-group had a porcelain aorta, versus none in the TF-group. More patients with critical preoperative state were included in the TA-group (TA:14\%, TF:2\%, $p=0.005)$ and the logistic Euro-SCORE was higher in the TA-group ( $36 \pm 15 \%$ for the TA-group compared to $25 \pm 4 \%$ for the TF-group; $p<0.001$ ).

Successful implantation rate was $100 \%$ with longer procedural time in the TF-group (TA:98 $\pm 33 \mathrm{~min}$, TF:127 \pm 56 min, $p<0.001)$. Twelve patients had redo

Table 3 Procedural data

\begin{tabular}{|c|c|c|c|c|}
\hline & $\begin{array}{l}\text { Overall } \\
(N=180)\end{array}$ & TA-TAVI $(N=90)$ & TF-TAVI $(N=90)$ & $p$ \\
\hline Sapien $^{\mathrm{TM}}$ and Sapien $\mathrm{XT}^{\mathrm{TM}}$ & $146(81 \%)$ & $86(96 \%)$ & $60(67 \%)$ & \\
\hline Sapien $3^{\text {TM }}$ & $8(4 \%)$ & $4(4 \%)$ & $4(4 \%)$ & \\
\hline CoreValve $^{T M}$ & $26(29 \%)$ & NA & $26(29 \%)$ & \\
\hline Valve-in-valve in degenerated bioprosthesis & $12(7 \%)$ & $7(8 \%)$ & $5(6 \%)$ & $0.550^{\mathrm{a}}$ \\
\hline Bailout valve-in-valve for migration or malpositioning & $7(4 \%)$ & $3(3 \%)$ & $4(4 \%)$ & 1 \\
\hline Mean valve size $(\mathrm{mm})$ & $25 \pm 2.1$ & $25 \pm 1.9$ & $25 \pm 2.2$ & \\
\hline \multicolumn{5}{|l|}{ Valve size distribution } \\
\hline $23 \mathrm{~mm}$ & $75(42 \%)$ & $40(44 \%)$ & $35(39 \%)$ & \\
\hline $26 \mathrm{~mm}$ & $77(43 \%)$ & $43(48 \%)$ & $44(49 \%)$ & \\
\hline $29 \mathrm{~mm}$ & $14(8 \%)$ & $7(8 \%)$ & $7(8 \%)$ & \\
\hline $31 \mathrm{~mm}$ & $4(4 \%)$ & $0(0 \%)$ & $4(4 \%)$ & \\
\hline Procedural time (min) & $113 \pm 48$ & $98 \pm 33$ & $127 \pm 56$ & $<0.001$ \\
\hline
\end{tabular}

Data presented as mean \pm SD or $\mathrm{N}(\%)$

${ }^{\text {a } C h i 2}$ value, otherwise it's a Fischer test or a T-Test value 
Table 4 Hospital outcome

\begin{tabular}{|c|c|c|c|c|}
\hline & $\begin{array}{l}\text { Overall } \\
(N=180)\end{array}$ & $\begin{array}{l}\text { TA-TAVI } \\
(N=90)\end{array}$ & $\begin{array}{l}\text { TF-TAVI } \\
(N=90)\end{array}$ & $p$ \\
\hline Hospital mortality & $17(9 \%)$ & $8(9 \%)$ & $9(10 \%)$ & $0.799^{*}$ \\
\hline \multicolumn{5}{|l|}{ Cause of death } \\
\hline Respiratory failure & $3(2 \%)$ & $3(3 \%)$ & $0(0 \%)$ & \\
\hline Cardiac tamponade for annulus rupture & $2(1 \%)$ & $0(0 \%)$ & $2(2 \%)$ & \\
\hline Valve migration & $1(1 \%)$ & $0(0 \%)$ & $1(1 \%)$ & \\
\hline Myocardial infarction & $1(1 \%)$ & $1(1 \%)$ & $0(0 \%)$ & \\
\hline Heart failure & $1(1 \%)$ & $0(0 \%)$ & $1(1 \%)$ & \\
\hline Sudden death & $1(1 \%)$ & $1(1 \%)$ & $0(0 \%)$ & \\
\hline Cardiac arrest & $2(1 \%)$ & $0(0 \%)$ & $2(2 \%)$ & \\
\hline Life-threatening bleeding & $1(1 \%)$ & $1(1 \%)$ & $0(0 \%)$ & \\
\hline Multiple organ failure & $1(1 \%)$ & $1(1 \%)$ & $0(0 \%)$ & \\
\hline Disabling stroke & $4(2 \%)$ & $1(1 \%)$ & $3(3 \%)$ & \\
\hline \multicolumn{5}{|l|}{ Complications } \\
\hline $\begin{array}{l}\text { Major vascular complications (including access related vascular complication, aortic } \\
\text { rupture and dissection, leg ischemia) }\end{array}$ & $13(7 \%)$ & $3(3 \%)$ & $10(11 \%)$ & 0.081 \\
\hline Valve migration & $3(2 \%)$ & $1(1 \%)$ & $2(2 \%)$ & 1 \\
\hline $\begin{array}{l}\text { Major/life-threatening bleeding (including cardiac tamponade for aortic rupture or } \\
\text { ventricular tear) }\end{array}$ & $7(4 \%)$ & $3(3 \%)$ & $4(4 \%)$ & 1 \\
\hline Disabling Stroke & $5(3 \%)$ & $2(2 \%)$ & $3(3 \%)$ & 1 \\
\hline Coronary occlusion & $1(1 \%)$ & $1(1 \%)$ & $0(0 \%)$ & 1 \\
\hline Bailout Sapien-in-Sapien for stent-valve migration or malpositioning & $7(4 \%)$ & $3(3 \%)$ & $4(4 \%)$ & 1 \\
\hline Pneumonia & $6(3 \%)$ & $5(6 \%)$ & $1(1 \%)$ & 0.211 \\
\hline Rethoracotomy for bleeding (TA)/pericardial drainage for tamponade (TF) & $5(3 \%)$ & $3(3 \%)$ & $2(2 \%)$ & 1 \\
\hline Postoperative acute renal failure & $4(2 \%)$ & $3(3 \%)$ & $1(1 \%)$ & 0.621 \\
\hline Dialysis & $3(2 \%)$ & $3(3 \%)$ & $0(0 \%)$ & 0.246 \\
\hline New pacemaker for conduction abnormality & $7(4 \%)$ & $2(2 \%)$ & $5(6 \%)$ & 0.444 \\
\hline Conversion to sternotomy & $2(1 \%)$ & $2(2 \%)$ & $0(0 \%)$ & 0.497 \\
\hline Bailout cardiopulmonary bypass & $4(2 \%)$ & $4(4 \%)$ & $0(0 \%)$ & 0.121 \\
\hline Early extubation & $123(68 \%)$ & $40(44 \%)$ & $83(92 \%)$ & $<0.001^{*}$ \\
\hline Intensive Care Unit stay (days) & $\begin{array}{l}1.4 \pm 4.1 \\
(\text { median:0) }\end{array}$ & $\begin{array}{l}2.6 \pm 5.5 \\
\text { (median:1) }\end{array}$ & $\begin{array}{l}0.2 \pm 0.7 \\
(\text { median:0) }\end{array}$ & $<0.001$ \\
\hline Hospital stay (days) & $\begin{array}{l}11.7 \pm 9.1 \\
\text { (median:9) }\end{array}$ & $\begin{array}{l}13.9 \pm 9.5 \\
\text { (median:10) }\end{array}$ & $\begin{array}{l}9.4 \pm 8.2 \\
\text { (median:8) }\end{array}$ & $<0.001$ \\
\hline Postoperative peak gradient (mmHg) & $17.6 \pm 9.5$ & $16.7 \pm 9.4$ & $18.5 \pm 9.5$ & 0.219 \\
\hline Postoperative mean gradient (mmHg) & $9.5 \pm 5.3$ & $9.2 \pm 5.1$ & $9.7 \pm 5.5$ & 0.563 \\
\hline Paravalvular leak: mild to severe & $28(16 \%)$ & $5(6 \%)$ & $23(26 \%)$ & $<0.001^{*}$ \\
\hline Trace of paravalvular leak & $48(27 \%)$ & 15 (17\%) & $33(37 \%)$ & \\
\hline Mild paravalvular leak & $25(14 \%)$ & $5(5 \%)$ & $20(22 \%)$ & \\
\hline Moderate paravalvular leak & $2(1 \%)$ & $0(0 \%)$ & $2(2 \%)$ & \\
\hline Severe paravalvular leak & $1(1 \%)$ & $0(0 \%)$ & $1(1 \%)$ & \\
\hline
\end{tabular}

Data presented as mean \pm SD or $\mathrm{N}(\%)$

${ }^{*}$ Chi2 value, otherwise it's a Fischer test or a T-Test value 


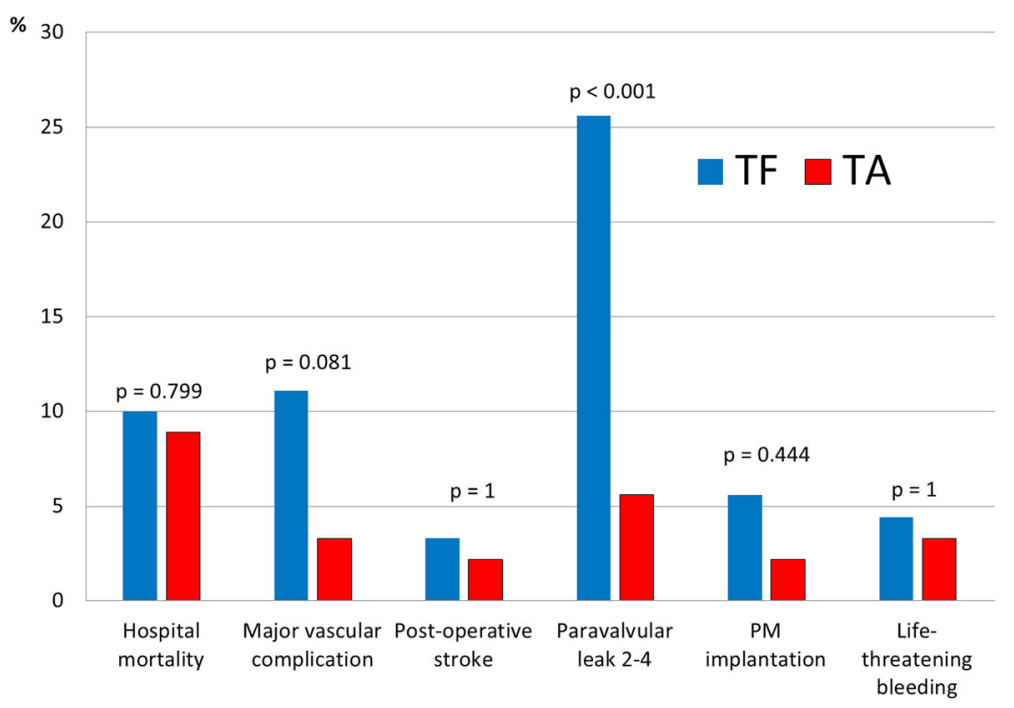

Fig. 2 Postoperative complications of TA and TF TAVI according to the VARC-2 definitions

valve-in-valve for degenerated aortic bioprosthesis, while seven patients received two stent-valves because of a stent-valve malpositioning or migration of the previously implanted stent-valve.

Mean valve size and valve size distribution are listed in Table 3.

With regards to hospital mortality and complication, variables were analysed using VARC-2 definitions (Table 4). Mortality for the two groups was similar (TA:9\%, TF:10\%, $p=0.799$ ) with a learning curve effect at the beginning of our experience. Main cause of death in TA-group was respiratory failure (3\%), whereas in the TFgroup was disabling stroke (3\%). Major vascular complications (including access-related vascular complication, aortic dissection, acute leg ischemia and aortic rupture) occurred more often in the TF-group (TA:3\%, TF:11\%, $p=0.081$ ) whereas major/life-threatening bleeding (including cardiac tamponade) (TA:3\%, TF:4\%, $p=1$ ), disabling stroke (TA:2\%, TF:3\%, $p=1$ ), and bailout valve-invalve for valve migration or malpositioning (TA:3\%, TF:4\%, $p=1$ ) were equally distributed (Fig. 2). Rethoracotomy for bleeding was performed in three TA and a percutaneous pericardial drainage for tamponade was urgently performed in two TF without further surgical exploration. Three patients in TA-group required dialysis.

As per the onset of new conduction abnormalities leading to pacemaker implantation, five devices were implanted in the TF-group ( 2 in Sapien ${ }^{\mathrm{TM}}$ valves and 3 in CoreValves $\left.^{\mathrm{Tm}}\right)$ and two in the TA-group $(p=0.444)$.

Echocardiographic controls showed similar peak (TA:17 $\pm 9.4 \mathrm{mmHg}$, TF: $18 \pm 9.5, \mathrm{mmHg} ; p=0.219$ ) and mean transvalvular gradients (TA:9.2 $\pm 5.1 \mathrm{mmHg}$, TF:9.7 $\pm 5.5 \mathrm{mmHg} ; p=0.563$ ) in both groups. Mild to severe paravalvular leaks were detected more in TF cases
(TA:5\%, TF:25\%, $p<0.001$ ) (Table 4). Nevertheless, moderate and severe paravalvular leaks were $0 \%$ in TA and $3 \%$ in TF patients.

Selected variables were analysed as risk factors for hospital mortality (Table 5), major vascular complications (Table 6) and disabling neurological complications according to the VARC-2 definitions (Table 7).

Concerning the hospital mortality, some variables were statistically related to a higher risk of death: critical preoperative state ( $p=0.026$; OR, 4.25; 95\% CI, 1.19-15.24), major vascular complications $(p=0.001$; OR, 8.07; 95\% CI, $2.28-28.53)$, stent-valve migration $(p=0.014$; OR, 21.6; 95\% CI, 1.85-252), life-threatening bleeding ( $p=0.001$; OR, 16.41; 95\% CI, 3.31-81.29), disabling stroke ( $p=0.003$; OR, 17.25; 95\% CI, 2.66-112), renal failure ( $p=0.022$; OR, 10.73; 95\% CI, 1.41-81.73), postoperative dialysis $(p=0.014$; OR, 21.6; 95\% CI, 1.85-252) and need for emergency cardiopulmonary bypass $(p=0.003$; OR, 34.71; $95 \%$ CI, 3.38-356) (Table 5). Early extubation (defined as extubation within $4 \mathrm{~h}$ since the end of the index procedure) represents a protective factor against hospital mortality ( $p=0.001$; OR, 0.16; 95\% CI, 0.05-0.48).

About risk factors for major vascular complications, use of emergency cardiopulmonary bypass was associated with higher rate of vascular injury $(p=0.010$; OR, 15; 95\% CI, 1.93-116) (Table 6). Concerning risk factors for disabling stroke, postoperative acute renal failure and dialysis were associated with poor neurological outcome ( $p=0.035$; OR, 14.33; 95\% CI, 1.21-169; and $p=0.020$; OR, 21.62; 95\% CI, 1.61-290, respectively) (Table 7).

\section{Discussion}

Since the beginning of transcatheter aortic valve therapies, the two main access routes are the 
Table 5 Univariate logistical regression analysis for hospital mortality (N:180)

\begin{tabular}{|c|c|c|c|c|c|c|c|c|c|}
\hline \multirow[t]{2}{*}{ Total } & \multirow{2}{*}{$\begin{array}{c}\text { n.0 } \\
163\end{array}$} & \multicolumn{8}{|l|}{ n.1 } \\
\hline & & 17 & & & & & & & \\
\hline Binary variables & n.o & p.0 & n.1 & p.1 & or & or.sd & or.ci95 & & $\mathrm{p}$ \\
\hline Transfemoral procedure & 81 & $49.7 \%$ & 9 & $52.9 \%$ & 1.14 & 1.67 & 0.42 & 3.10 & 0.799 \\
\hline Gender (M) & 76 & $46.6 \%$ & 6 & $35.3 \%$ & 0.62 & 1.70 & 0.22 & 1.77 & 0.375 \\
\hline COPD & 34 & $20.9 \%$ & 4 & $23.5 \%$ & 1.17 & 1.83 & 0.36 & 3.81 & 0.798 \\
\hline Vascular disease & 81 & $49.7 \%$ & 10 & $58.8 \%$ & 1.45 & 1.68 & 0.52 & 3.98 & 0.476 \\
\hline Previous vascular surgery & 16 & $9.8 \%$ & 1 & $5.9 \%$ & 0.57 & 2.90 & 0.07 & 4.62 & 0.602 \\
\hline Coronary disease & 82 & $50.3 \%$ & 8 & $47.1 \%$ & 0.88 & 1.67 & 0.32 & 2.39 & 0.799 \\
\hline Previous CABG & 25 & $15.3 \%$ & 2 & $11.8 \%$ & 0.74 & 2.19 & 0.16 & 3.42 & 0.696 \\
\hline Previous cardiac surgery & 35 & $21.5 \%$ & 5 & $29.4 \%$ & 1.52 & 1.76 & 0.50 & 4.62 & 0.456 \\
\hline Previous STENT & 23 & $14.1 \%$ & 5 & $29.4 \%$ & 2.54 & 1.78 & 0.82 & 7.87 & 0.107 \\
\hline Hypertension & 108 & $66.3 \%$ & 9 & $52.9 \%$ & 0.57 & 1.67 & 0.21 & 1.57 & 0.278 \\
\hline Renal failure & 66 & $40.5 \%$ & 9 & $52.9 \%$ & 1.65 & 1.67 & 0.61 & 4.51 & 0.326 \\
\hline Previous stroke & 17 & $10.4 \%$ & 3 & $17.6 \%$ & 1.84 & 1.99 & 0.48 & 7.06 & 0.374 \\
\hline Diabetes & 27 & $16.6 \%$ & 3 & $17.6 \%$ & 1.08 & 1.95 & 0.29 & 4.01 & 0.909 \\
\hline Pacemaker & 19 & $11.7 \%$ & 2 & $11.8 \%$ & 1.01 & 2.21 & 0.21 & 4.77 & 0.989 \\
\hline Thorax radiotherapy & 14 & $8.6 \%$ & 2 & $11.8 \%$ & 1.42 & 2.23 & 0.29 & 6.85 & 0.663 \\
\hline Critical state & 11 & $6.7 \%$ & 4 & $23.5 \%$ & 4.25 & 1.92 & 1.19 & 15.24 & 0.026 \\
\hline Logistic EuroScore > 20\% & 117 & $71.8 \%$ & 12 & $70.6 \%$ & 0.94 & 1.75 & 0.31 & 2.83 & 0.917 \\
\hline LVEF > 50\% & 91 & $55.8 \%$ & 10 & $62.5 \%$ & 1.32 & 1.72 & 0.46 & 3.80 & 0.608 \\
\hline Pulmonary hypertension & 95 & $58.3 \%$ & 6 & $35.3 \%$ & 0.39 & 1.70 & 0.14 & 1.11 & 0.077 \\
\hline Valve-in-valve & 11 & $6.7 \%$ & 1 & $5.9 \%$ & 0.86 & 2.94 & 0.10 & 7.13 & 0.892 \\
\hline Vascular complication & 8 & $4.9 \%$ & 5 & $29.4 \%$ & 8.07 & 1.90 & 2.28 & 28.53 & 0.001 \\
\hline Valve migration & 1 & $0.6 \%$ & 2 & $11.8 \%$ & 21.60 & 3.50 & 1.85 & 252 & 0.014 \\
\hline Life-threatening bleeding & 3 & $1.8 \%$ & 4 & $23.5 \%$ & 16.41 & 2.26 & 3.31 & 81.29 & 0.001 \\
\hline Postoperative stroke & 2 & $1.2 \%$ & 3 & $17.6 \%$ & 17.25 & 2.60 & 2.66 & 112 & 0.003 \\
\hline Pneumonia & 4 & $2.5 \%$ & 2 & $11.8 \%$ & 5.30 & 2.48 & 0.90 & 31.37 & 0.066 \\
\hline Postoperative acute renal failure & 2 & $1.2 \%$ & 2 & $11.8 \%$ & 10.73 & 2.82 & 1.41 & 81.73 & 0.022 \\
\hline Postoperative dialysis & 1 & $0.6 \%$ & 2 & $11.8 \%$ & 21.60 & 3.50 & 1.85 & 252 & 0.014 \\
\hline New pacemaker & 6 & $3.7 \%$ & 1 & $5.9 \%$ & 1.64 & 3.04 & 0.19 & 14.45 & 0.658 \\
\hline Cardiopulmonary bypass use & 1 & $0.6 \%$ & 3 & $17.6 \%$ & 34.71 & 3.28 & 3.38 & 356 & 0.003 \\
\hline Early extubation (within 4 h) & 118 & $72.4 \%$ & 5 & $29.4 \%$ & 0.16 & 1.75 & 0.05 & 0.48 & 0.001 \\
\hline Paravalvular leak (mild to severe) & 25 & $15.4 \%$ & 3 & $20.0 \%$ & 1.37 & 1.98 & 0.36 & 5.21 & 0.644 \\
\hline
\end{tabular}

OR odds ratio, OR.SD odds ratio standard deviation, OR.CI95 odds ratio 95\% confidence interval, COPD chronic obstructive pulmonary disease, CABG coronary artery bypass graft, LVEF left ventricular ejection fraction

transfemoral and the transapical ones, allowing for placement of balloon-expandable and self-expanding valves of different size. However, the allocation to one of these two access routes is still questionable and based, at the time being, on the presence of a severe vascular disease of the aorta or ilio-femoral vessels, or on the possibility of performing the TF case under sedation [18-22].

In our experience, patients allocated to the two groups (all performed under general anaesthesia) showed different comorbidity patterns: patients in the TA-group presented higher prevalence of comorbidities, higher logistic Euro-SCORE and were more often in a preoperative critical state. Thus, according to the fact that all patients with severe vascular disease were automatically included in the TA-group, we faced a TA population carrying a higher risk profile compared to the TF population. However, the hospital mortality rate and neurological outcome were similar.

Hospital mortality of our first TAVI series is in-line with published preliminary results of TA vs TF showing rates ranges between $5 \%$ and $15 \%$, without statistical 
Table 6 Univariate logistical regression analysis for major vascular complications (N:180)

\begin{tabular}{|c|c|c|c|c|c|c|c|c|c|}
\hline \multirow{3}{*}{$\begin{array}{l}\text { Total } \\
\text { Binary variables }\end{array}$} & \multirow{3}{*}{$\begin{array}{l}\text { n.0 } \\
167 \\
\text { n.0 }\end{array}$} & \multicolumn{8}{|l|}{ n.1 } \\
\hline & & \multicolumn{8}{|l|}{13} \\
\hline & & p.0 & $\overline{\mathrm{n} .1}$ & p.1 & or & or.sd & or.ci95 & & $p$ \\
\hline Transfemoral procedure & 80 & $47.9 \%$ & 10 & $76.9 \%$ & 3.62 & 1.97 & 0.96 & 13.64 & 0.057 \\
\hline Gender (M) & 78 & $46.7 \%$ & 4 & $30.8 \%$ & 0.51 & 1.86 & 0.15 & 1.71 & 0.274 \\
\hline COPD & 36 & $21.6 \%$ & 2 & $15.4 \%$ & 0.66 & 2.21 & 0.14 & 3.12 & 0.602 \\
\hline Vascular disease & 84 & $50.3 \%$ & 7 & $53.8 \%$ & 1.15 & 1.78 & 0.37 & 3.58 & 0.806 \\
\hline Previous vascular surgery & 15 & $9.0 \%$ & 2 & $15.4 \%$ & 1.84 & 2.26 & 0.37 & 9.10 & 0.453 \\
\hline Coronary disease & 85 & $50.9 \%$ & 5 & $38.5 \%$ & 0.60 & 1.81 & 0.19 & 1.92 & 0.392 \\
\hline Previous CABG & 26 & $15.6 \%$ & 1 & $7.7 \%$ & 0.45 & 2.89 & 0.06 & 3.62 & 0.455 \\
\hline Previous cardiac surgery & 38 & $22.8 \%$ & 2 & $15.4 \%$ & 0.62 & 2.20 & 0.13 & 2.91 & 0.542 \\
\hline Hypertension & 110 & $65.9 \%$ & 7 & $53.8 \%$ & 0.60 & 1.79 & 0.19 & 1.88 & 0.385 \\
\hline Renal failure & 72 & $43.1 \%$ & 3 & $23.1 \%$ & 0.40 & 1.97 & 0.11 & 1.49 & 0.171 \\
\hline Diabetes & 29 & $17.4 \%$ & 1 & $7.7 \%$ & 0.40 & 2.89 & 0.05 & 3.17 & 0.383 \\
\hline Pacemaker implantation & 20 & $12.0 \%$ & 1 & $7.7 \%$ & 0.61 & 2.91 & 0.08 & 4.97 & 0.646 \\
\hline Thorax radiotherapy & 14 & $8.4 \%$ & 2 & $15.4 \%$ & 1.99 & 2.27 & 0.40 & 9.87 & 0.401 \\
\hline Critical state & 14 & $8.4 \%$ & 1 & $7.7 \%$ & 0.91 & 2.94 & 0.11 & 7.53 & 0.931 \\
\hline Logistic EuroSCORE $>20 \%$ & 121 & $72.5 \%$ & 8 & $61.5 \%$ & 0.61 & 1.81 & 0.19 & 1.96 & 0.404 \\
\hline LVEF $>50 \%$ & 95 & $57.2 \%$ & 6 & $46.2 \%$ & 0.64 & 1.78 & 0.21 & 1.99 & 0.441 \\
\hline Pulmonary hypertension & 96 & $57.5 \%$ & 5 & $38.5 \%$ & 0.46 & 1.81 & 0.15 & 1.47 & 0.192 \\
\hline Valve-in-valve & 11 & $6.6 \%$ & 1 & $7.7 \%$ & 1.18 & 2.96 & 0.14 & 9.94 & 0.878 \\
\hline Hospital mortality & 12 & $7.2 \%$ & 5 & $38.5 \%$ & 8.07 & 1.90 & 2.28 & 28.53 & 0.001 \\
\hline Disabling Stroke & 4 & $2.4 \%$ & 1 & $7.7 \%$ & 3.40 & 3.18 & 0.35 & 32.82 & 0.291 \\
\hline Pericardial drainage/rethoracotomy for bleeding & 4 & $2.4 \%$ & 1 & $7.7 \%$ & 3.40 & 3.18 & 0.35 & 32.82 & 0.291 \\
\hline Postoperative acute renal failure & 3 & $1.8 \%$ & 1 & $7.7 \%$ & 4.56 & 3.30 & 0.44 & 47.19 & 0.204 \\
\hline Postoperative dialysis & 2 & $1.2 \%$ & 1 & $7.7 \%$ & 6.87 & 3.53 & 0.58 & 81.36 & 0.126 \\
\hline Conversion to sternotomy & 1 & $0.6 \%$ & 1 & $7.7 \%$ & 13.83 & 4.24 & 0.81 & 235 & 0.069 \\
\hline Cardiopulmonary bypass use & 2 & $1.2 \%$ & 2 & $15.4 \%$ & 15.00 & 2.85 & 1.93 & 116 & 0.010 \\
\hline Early extubation (within 4 h) & 117 & $70.1 \%$ & 6 & $46.2 \%$ & 0.37 & 1.79 & 0.12 & 1.14 & 0.084 \\
\hline Paravalvular leak (mild to severe) & 26 & $15.8 \%$ & 2 & $16.7 \%$ & 1.07 & 2.23 & 0.22 & 5.16 & 0.934 \\
\hline
\end{tabular}

OR odds ratio, OR.SD odds ratio standard deviation, OR.CI95 odds ratio $95 \%$ confidence interval, COPD chronic obstructive pulmonary disease, CABG coronary artery bypass graft, LVEF left ventricular ejection fraction

differences between the groups [18-22]. In a retrospective study, Van der Boon reported hospital mortality of $6.4 \%$ for $\mathrm{TF}$ and $15.7 \%$ for TA whereas in a prospective study including thousand TAVI from Schymik and al. the mortality was $6.5 \%$ for TF and $6.1 \%$ for TA $[19,21]$. Murarka and al. also reported promising mortality rates of $4.5 \%$ for $\mathrm{TF}$ and $5.3 \%$ for TA in a retrospective singlecentre experience [22]. However, none of these three aforementioned studies was able to demonstrate a statistically significant difference between the two popular approaches.

In our report, neurological outcome showed similar results for TA and TF (stroke $=$ TA:2\%, TF:3\%, $p=1$ ) and we didn't observe a significant difference. Our results are similar to published data. Schymik described
$2.3 \%$ of stroke in the TF-group and $1.7 \%$ in the TAgroup with a non-significant $p$-value between the groups. Other published series show similar conclusions [18-22].

If we consider paravalvular leaks and access-related vascular injuries, we identified a discrepancy in favour for the TA approach. In particular, the mild to severe paravalvular leak incidence (TA:6\%, TF:26\%, $p<0.001$ ) of our patients was similar to the one published in a retrospective study from Greason and co-workers who reported $12 \%$ of mild to severe paravalvular leak in TF versus $8.4 \%$ in the TA group [18]. Nevertheless, the incidence of moderate-severe PVL only is low in our groups and almost similar between the two population. Murarka and colleagues as well, have observed similar results 
Table 7 Univariate logistical regression analysis for neurological complications (N:180)

\begin{tabular}{|c|c|c|c|c|c|c|c|c|c|}
\hline & n.0 & n.1 & & & & & & & \\
\hline Total & 175 & 5 & & & & & & & \\
\hline Binary variables & n.0 & p.0 & n.1 & p.1 & or & or.sd & \multicolumn{2}{|c|}{ or.ci95 } & $\mathrm{p}$ \\
\hline Transfemoral procedure & 87 & $49.7 \%$ & 3 & $60.0 \%$ & 1.52 & 2.52 & 0.25 & 9.30 & 0.652 \\
\hline Gender (M) & 81 & $46.3 \%$ & 1 & $20.0 \%$ & 0.29 & 3.09 & 0.03 & 2.65 & 0.273 \\
\hline COPD & 37 & $21.1 \%$ & 1 & $20.0 \%$ & 0.93 & 3.11 & 0.10 & 8.59 & 0.951 \\
\hline Vascular disease & 88 & $50.3 \%$ & 3 & $60.0 \%$ & 1.48 & 2.52 & 0.24 & 9.09 & 0.670 \\
\hline Coronary disease & 88 & $50.3 \%$ & 2 & $40.0 \%$ & 0.66 & 2.52 & 0.11 & 4.04 & 0.652 \\
\hline Previous CABG & 26 & $14.9 \%$ & 1 & $20.0 \%$ & 1.43 & 3.12 & 0.15 & 13.33 & 0.752 \\
\hline Previous cardiac surgery & 38 & $21.7 \%$ & 2 & $40.0 \%$ & 2.40 & 2.54 & 0.39 & 14.91 & 0.346 \\
\hline Previous coronary stenting & 27 & $15.4 \%$ & 1 & $20.0 \%$ & 1.37 & 3.12 & 0.15 & 12.74 & 0.782 \\
\hline Hypertension & 114 & $65.1 \%$ & 3 & $60.0 \%$ & 0.80 & 2.53 & 0.13 & 4.93 & 0.812 \\
\hline Previous stroke & 19 & $10.9 \%$ & 1 & $20.0 \%$ & 2.05 & 3.14 & 0.22 & 19.33 & 0.530 \\
\hline Diabetes & 29 & $16.6 \%$ & 1 & $20.0 \%$ & 1.26 & 3.12 & 0.14 & 11.67 & 0.840 \\
\hline LVEF $>50 \%$ & 97 & $55.7 \%$ & 4 & $80.0 \%$ & 3.18 & 3.09 & 0.35 & 28.99 & 0.306 \\
\hline Pulmonary hypertension & 98 & $56.0 \%$ & 3 & $60.0 \%$ & 1.18 & 2.52 & 0.19 & 7.23 & 0.859 \\
\hline Hospital mortality & 14 & $8.0 \%$ & 3 & $60.0 \%$ & 17.25 & 2.60 & 2.66 & 112 & 0.003 \\
\hline Vascular complication & 12 & $6.9 \%$ & 1 & $20.0 \%$ & 3.40 & 3.18 & 0.35 & 32.82 & 0.291 \\
\hline Postoperative acute renal failure & 3 & $1.7 \%$ & 1 & $20.0 \%$ & 14.33 & 3.53 & 1.21 & 169 & 0.035 \\
\hline Postoperative dialysis & 2 & $1.1 \%$ & 1 & $20.0 \%$ & 21.62 & 3.76 & 1.61 & 290 & 0.020 \\
\hline Early extubation (within 4 h) & 121 & $69.1 \%$ & 2 & $40.0 \%$ & 0.30 & 2.53 & 0.05 & 1.83 & 0.191 \\
\hline
\end{tabular}

OR odds ratio, OR.SD odds ratio standard deviation, OR.CI95 odds ratio $95 \%$ confidence interval, COPD chronic obstructive pulmonary disease, CABG coronary artery bypass grafting, LVEF left ventricular ejection fraction

between TA and TF with $7.6 \%$ of moderate-severe paravalvular leaks in TF-group and $7 \%$ in TA-group $(p=0.999)$ [22]. The reason for some discrepancies in paravalvular leak rate in the 2 techniques can be due to the more direct and more precise valve positioning during TA cases but this is not proved yet. Moreover, as far as paravalvular leaks are concerned, we have to admit that, in our experience, we have seen a great improvement after the launch of last-generation Sapien ${ }^{\text {тм }} 3$ valve featuring an innovative outer skirt preventing leaks. Therefore, we can speculate that, in the future, the use of this valve (or other stent-valves addressing the issue of paravalvular leaks with improved technologies) will reduce the incidence of clinically-relevant leaks.

Regarding the major access-related vascular complication rate in our group, this was higher in TF patients but the result is not statistically significant (TA:3\%, TF:11\%, $p=0.081$ ). In comparison, if we look at recent publications we can see that the incidence of this complication is more prevalent in TF as well: Schymik reported $17.5 \%$ of major vascular complications in TF versus $2.5 \%$ in TA ( $p=<0.0001)$ [19]. Murarka and colleagues observed similar trend, with $12.1 \%$ of major vascular complications in the TFgroup versus $0 \%$ in the TA-group [22]. New generation devices with low-profile introducer sheaths and small delivery systems will help decreasing the incidence of access site vascular complications in future reports.

As long as the intensive care unit length of stay and extubation time are concerned, in our study these numbers were slightly longer for TA-TAVI patients and this finding is justified by the fact that at the beginning TA patients were all transferred, intubated, to the intensive care unit and then transferred to the intermediate care unit on postoperative day one. On the other hand, since the beginning, complication-free transfemoral TAVI cases were rapidly extubated in the cath-lab and transferred to the intermediate care unit. However, these results are not comparable with other centres as local hospital policies and daily practice can be very different and, sometimes, they can change with acquired experience.

An important point of discussion is the development of new TAVI devices: new generations of stent-valve equipment have more performant designs and lowprofile delivery systems assuring lower incidence of paravalvular leak and vascular injury. This development can have a great impact in future reports on TAVI mortality and morbidity allowing for the use of these new devices in mid-risk and younger patients.

Our study presents some limitations. It is a retrospective study describing the hospital outcome of first cases 
of TA and TF TAVI performed at our Institution with a relatively small number of patients. The population included the preliminary TAVI experience of our hospital and, therefore, a physiologic learning curve can have negatively affected the first-period clinical outcome. The patient-selection process has also changed during the study period as well as the use of new-generation devices.

\section{Conclusion}

In conclusion, this study shows our first TAVI experience and results are similar to published data. Based on our findings, we can confirm that transcatheter techniques are promising and provide good hospital outcomes. Improved surgeons' and cardiologists' skills and the advent of last-generation devices will facilitate the stent-valve delivery and will help improving the risk of vascular complication, neurological complication and paravalvular leak, also for use in intermediate-risk patients.

\section{Abbreviations}

3D-CT scan: Three-dimensional Computed Tomography scan; COPD: Chronic obstructive pulmonary disease; LVEF: Left ventricular ejection fraction; SAVR: Standard aortic valve replacement; TA: Transapical; TAVI: Transcatheter aortic valve implantation; TF: Transfemoral; VARC: Valve Academic Research Consortium

\section{Acknowledgments}

We thank Jérôme Pasquier, statistician at University of Lausanne, Lausanne, Switzerland.

\section{Funding}

No founding was provided.

\section{Availability of data and materials}

Please contact the corresponding author for data requests.

\section{Authors' contributions}

EF: conceived of the study, wrote the manuscript, analysed data, study design, performed procedures. EE: collected data, performed procedures. SK: collected data, analysed data, wrote part of the manuscript. OM: collected data, performed procedures. PT: study design, revised the manuscript. DB: collected data, participated in TAVI procedures, revised the manuscript. LvS: conceived of the study, supervision. All authors read and approved the final manuscript.

\section{Ethics approval and consent to participate}

The patients inclusion in the Swiss TAVI Registry has been approved by the Ethics Committee of the Vaud State in Switzerland. All patients approved and signed informed consent for surgery and use of data for clinical research.

\section{Consent for publication}

All patients approved and signed informed consents for surgery and use of data for clinical research (Swiss TAVI Registry).

\section{Competing interests}

Dr. Enrico Ferrari is consultant and proctor for Edwards Lifesciences. Other Authors have no conflicts of interest.

\section{Publisher's Note}

Springer Nature remains neutral with regard to jurisdictional claims in published maps and institutional affiliations.

\section{Author details}

'Department of Cardiac Surgery, Cardiocentro Ticino Foundation, Via Tesserete 48, 6900 Lugano, Switzerland. ${ }^{2}$ Cardiovascular Research Unit, University Hospital, Lausanne, Switzerland. ${ }^{3}$ Cardiology Unit, University Hospital, Lausanne, Switzerland. ${ }^{4}$ Cardiac Surgery Unit, University Hospital of Basel, Basel, Switzerland.

Received: 11 January 2017 Accepted: 27 August 2017

Published online: 06 September 2017

\section{References}

1. Carabello BA. Aortic Stenosis. N Engl J Med. 2002;346:677-82.

2. Nishimura RA, Otto CM, Bonow RO, Carabello BA, Erwin JP III, Guyton RA, et al. 2014 AHA/ACC guideline for the management of patients with valvular heart disease: a report of the American College of Cardiology/ American Heart Association task force on practice guidelines. J Thorac Cardiovasc Surg. 2014;148:e1-e132.

3. Czarny MJ, Resar JR. Diagnosis and management of valvular aortic stenosis. Clin Med Insights Cardiol. 2014:8(Suppl 1):15-24.

4. lung B, Baron G, Butchart EG, Delahaye F, Gohlke-Bärwolf C, Levang OW, et al. A prospective survey of patients with valvular heart disease in Europe: the euro heart survey on Valvular heart disease. Eur Heart 2003;24:1231-43.

5. Ferrari $E$, von Segesser LK. Transcatheter aortic valve implantation (TAVI): state of the art techniques and future perspectives. Swiss Med Wkly. 2010; 140:w13127. https://doi.org/10.4414/smw.2010.13127.

6. Ferrari E, Namasivayam J, Marcucci C, Gronchi F, Berdajs D, Niclauss L, von Segesser LK. Transapical aortic valve replacement in extreme-risk patients: outcome, risk factors and mid-term results. Eur J Cardiothorac Surg. 2013;43: 978-85.

7. Leon MB, Smith CR, Mack M, Miller DC, Moses JW, Svensson LG, et al. Transcatheter aortic-valve implantation for aortic stenosis in patients who cannot undergo surgery. N Engl J Med. 2010;363:1597-607.

8. Smith CR, Leon MB, Mack MJ, Miller DC, Moses JW, Svensson LG, et al. Transcatheter versus surgical aortic-valve replacement in high-risk patients. N Engl J Med. 2011;364:2187-98.

9. Codner P, Assali A, Dvir D, Vaknin-Assa H, Porat E, Shapira Y, et al. Two-year outcomes for patients with severe symptomatic aortic stenosis treated with transcatheter aortic valve implantation. Am J Cardiol. 2013;111:1330-6.

10. D'Onofrio A, Salizzoni S, Agrifoglio M, Cota L, Luzi G, Tartara PM, et al. Medium term outcomes of transapical aortic valve implantation: results from the Italian registry of trans-apical aortic valve implantation. Ann Thorac Surg. 2013;96:830-5

11. Schymik G, Schröfel H, Schymik JS, Wondraschek R, Süselbeck T, Kiefer R, et al. Acute and late outcomes of Transcatheter aortic valve implantation (TAVI) for the treatment of severe symptomatic aortic stenosis in patients at high- and low-surgical risk. J Intervent Cardiol. 2012;25:364-74.

12. Gotzmann $M$, Korten $M$, Bojara W, Lindstaedt $M$, Rahlmann $P$, Mügge $A$ et al. Long-term outcome of patients with moderate and severe prosthetic aortic valve regurgitation after transcatheter aortic valve implantation. Am J Cardiol. 2012;110:1500-6.

13. Ussia GP, Barbanti M, Cammalleri V, Scarabelli M, Mulè M, Aruta P, et al. Quality-of-life in elderly patients one year after transcatheter aortic valve implantation for severe aortic stenosis. Eurolntervention J Eur Collab Work Group Interv Cardiol Eur Soc Cardiol. 2011;7:573-9.

14. Georgiadou P, Kontodima P, Sbarouni E, Karavolias GK, Smirli A, Xanthos T, et al. Long-term quality of life improvement after transcatheter aortic valve implantation. Am Heart J. 2011;162:232-7.

15. Gonçalves A, Marcos-Alberca P, Almeria C, Feltes G, Hernández-Antolín RA Rodríguez E, et al. Quality of life improvement at midterm follow-up after transcatheter aortic valve implantation. Int J Cardiol. 2013;162:117-22.

16. Kappetein AP, Head SJ, Généreux P, Piazza N, van Mieghem NM, Blackstone $\mathrm{EH}$, et al. Valve academic research consortium-2. Updated standardized endpoint definitions for transcatheter aortic valve implantation: the valve academic research consortium-2 consensus document. J Thorac Cardiovasc Surg. 2013;145:6-23.

17. Ferrari E, Sulzer C, Marcucci C, Rizzo E, Tozzi P, von Segesser LK. Transapical aortic valve implantation without angiography - proof of concept. Ann Thorac Surg. 2010;89:1925-32.

18. Greason KL, Suri RM, Nkomo VT, Rihal CS, Holmes DR, Mathew V. Beyond the learning curve: transapical versus transfemoral transcatheter aortic valve 
replacement in the treatment of severe aortic valve stenosis. J Card Surg. 2014;29:303-7.

19. Van der Boon RMA, Marcheix B, Tchetche D, Chieffo A, Van Mieghem NM, Dumonteil $N$, et al. Transapical versus transfemoral aortic valve implantation: a multicenter collaborative study. Ann Thorac Surg. 2014;97:22-8.

20. Gaasch WH, D'Agostino RS. Transcatheter aortic valve implantation: the transfemoral versus the transapical approach. Ann Cardiothorac Surg. 2012; $1: 200-5$.

21. Schymik $G$, Würth $A$, Bramlage $P$, Herbinger $T$, Heimeshoff M, Pilz $L$, et al Long-term results of transapical versus transfemoral TAVI in a real world population of 1000 patients with severe symptomatic aortic stenosis. Circ Cardiovasc Interv. 2014;8(1):e000761. doi:10.1161/CIRCINTERVENTIONS.113. 000761

22. Murarka S, Lazkani M, Neihaus M, Boggess M, Morris M, Gellert G, et al. Comparison of 30-day outcomes of Transfemoral versus Transapical approach for Transcatheter aortic valve replacement: a single-center US experience. Ann Thorac Surg. 2015;99:1539-44.

Submit your next manuscript to BioMed Central and we will help you at every step:

- We accept pre-submission inquiries

- Our selector tool helps you to find the most relevant journal

- We provide round the clock customer support

- Convenient online submission

- Thorough peer review

- Inclusion in PubMed and all major indexing services

- Maximum visibility for your research

Submit your manuscript at www.biomedcentral.com/submit
Biomed Central 